\title{
- Faut-il craindre une pénurie des ressources en eau au XXIe siècle ? Les thèses en présence
}

\author{
— Towards Water Scarcity in the 21st Century? Current Schools of Thought -
}

\author{
par Jacques Labre \\ SAFEGE Ingénieurs Conseils
}

\section{Va-t-on manquer d'eau au siècle prochain ? Plusieurs thèses s'affrontent, certaines catastro- phistes, d'autres proposant des solutions pour une meilleure répartition des ressources.}

Plus encore que les précédentes, l'année 1997 a vu se succéder de nombreux colloques, congrès et forums internationaux sur le thème de la gestion des eaux dans un contexte de pénurie croissante ; en particulier s'est tenu à Montréal le IX $\mathrm{IX}^{\mathrm{e}}$ Congrès de l'Association Internationale des Ressources en Eau. Largement relayés par les médias, ces événements révèlent - et entretiennent - dans le public une inquiétude diffuse, celle d'une prochaine raréfaction des ressources en eau indispensables à la vie de l'homme et à la préservation des écosystèmes.

Par la voix du président Chirac, la France a proposé d'organiser une Conférence Internationale sur ce thème en 1998. Celle-ci sera-t-elle l'occasion de décliner à nouveau un " prêt à penser " générateur d'inquiétudes parfois contre-productives, ou de contribuer à une réelle hiérarchisation des enjeux et à des actions concrètes pour le " développement durable" ?

Les conditions préalables à un débat utile sur les ressources en eau sont :

- une bonne compréhension des thèses en présence aujourd'hui,

- la clarification des ambiguïtés sous-jacentes au discours de beaucoup de leaders d'opinion.

La crainte excessive d'une pénurie latente peut aussi causer des effets pervers pour l'ouverture des services de l'eau au secteur privé. Ce dernier n'a rien à gagner de la montée d'une sorte de dogmatisme, qui donnerait à l'industrie de l'eau une telle visibilité politique qu'elle deviendrait la cible privilégiée d'affrontements idéologiques.

Les positions actuellement en présence, peuvent être schématiquement réunies dans quatre familles de pensée, qui se sont toutes exprimées au Congrès de Montréal.

This article describes current schools of thought on water as a finite resource and, as such, a factor to be contended within the pursuit of sustainable development. The media, in inflating this topic, has tended to create stereotypes in the minds of the public and the leaders of opinion. These issues must be put into their proper perspective if a constructive debate is to ensue.

The four main schools of thought, addressed schematically in this article, are as follows:

- the neo-Malthusian school, which focuses on water as a key factor in global food security and asserts that, overall, the continued growth of man-made pressures on the hydrological cycle cannot fail to have adverse consequences for Biodiversity;

- the Planning-centred approach which places the emphasis on water resources as an aspect of economic development at the state level,

- the Geostrategic viewpoint, which stresses transboundary issues and the vulnerability of certain nations due to their lack of autonomy regarding water resources,

- the Economy-centred theory which advocates market mechanisms as a means of regulating resource allocation. This works by conciliating the interests of the individual with those of the economic community through instruments such as fees, incentives and water right transactions. 


\section{E LA THÈSE NÉO-MALTHUSIENNE}

Le postulat de cette thèse est que l'eau constituera au cours des 30 prochaines années le principal facteur limitant dans le développement de la production alimentaire. Elle s'appuie sur les travaux d'hydrologues et d'agronomes (Falkenmark, Shiklomanov ...) et sur des études de la FAO, selon lesquelles :

- une grande partie des besoins alimentaires nouveaux induits par la croissance démographique devra être satisfaite par l'agriculture irriguée,

- un nombre croissant de pays ne pourra accéder à l'autosuffisance alimentaire en raison de la disponibilité limitée en eau d'irrigation.

Effectivement, l'agriculture joue un rôle déterminant dans les bilans d'utilisation des eaux : on estime que dans le monde, elle est responsable des deux tiers des prélèvements dans les rivières et aquifères ; ce taux passe à $86 \%$ si on raisonne en consommation définitive, car contrairement aux autres usages, l'irrigation ne restitue au milieu naturel qu'une faible proportion des eaux prélevées. L'irrigation est la cause du tarissement des cours aval de fleuves comme le Nil (dont le débit moyen à l'embouchure est passé de 40 à $5 \mathrm{~km}^{3} / \mathrm{an}$ au cours des 30 dernières années), ou le Fleuve Jaune en saison sèche. Elle est aussi la cause du retrait de la Mer d'Aral ou de la Mer Morte.

Mais les avis des prévisionnistes diffèrent très fortement selon les auteurs :

- la situation actuelle, état zéro des projections, est très mal connue, en termes de surfaces irriguées comme de consommation d'eau ;

- la contribution relative de l'agriculture en sec et de l'agriculture irriguée à la production agricole future varie selon les experts (actuellement, les terres irriguées fournissent $40 \%$ de la recolte) ;

- l'efficience de l'eau employée aujourd'hui en irrigation est très médiocre (moins de $50 \%$ ). Il est techniquement possible d'atteindre $80 \%$. Le rythme d'amélioration escompté divise également les agronomes. Une chose est certaine : des investissements considérables seraient nécessaires pour y parvenir et il sera souvent difficile de les justifier économiquement au vu des seuls bénéfices pour l'agriculture ;

- les résultats des projections sont très sensibles aux hypothèses concernant le régime alimentaire des humains ; en effet il ne faut que 5001 d'eau pour produire $1 \mathrm{~kg}$ de pommes de terre, mais il en faut 20 à 100000 pour produire $1 \mathrm{~kg}$ de bœuf à partir de fourrages irrigués ;

- enfin, il y a de grandes divergences sur les prévisions démographiques à l'horizon considéré (2025).

Tous les auteurs s'accordent sur l'existence d'un " triangle de la pénurie " entre la Tunisie, le Soudan et le Pakistan. Dans cette région (et aussi dans quelques Etats insulaires de faible superficie), l'autosuffisance alimentaire sera hydrologiquement impossible à l'horizon 2025 (Seckler, 1997).
Pour le reste de la planète, les avis divergent fortement. La possibilité globale de nourrir l'humanité dans 2 générations n'est contestée que par les plus pessimistes. Falkenmark pense que la ressource en eau disponible pourrait compromettre l'autosuffisance alimentaire de pays regroupant la moitié de la population mondiale dans 30 ans.

S'appuyant sur ces projections qui restent très incertaines, on voit apparaître une école " néo-malthusianiste ", à l'approche radicale, qui globalise à l'échelle planétaire toutes les consommations d'eau pluviale ou d'eau libre imputables aux activités humaines (S. Postel, 1996). Si on cumule l'évaporation des cultures, des forêts utilisées par l'homme, des plans d'eau artificiels et des villes, l'homme s'approprierait déjà $1 / 4$ de l'évaporation terrestre totale, qui ferait défaut aux écosystèmes naturels. La pression actuelle étant considérée comme le maximum écologiquement admissible, il conviendrait de geler le niveau des prélèvements. C'est cette école, bien représentée aux USA et dans les pays nordiques, qui est à l'origine du lobby " anti-barrages " très actif auprès des institutions internationales. Elle considère tout barrage comme intrinsèquement néfaste au motif qu'il contribue à accroitre la part du cycle de l'eau " détournée " par l'homme.

\section{II — LA THÈSE DU PLANIFICATEUR : L'EAU, THÈME D'AMÉNAGEMENT DU TERRITOIRE}

A l'opposé de ce point de vue globalisant, les Etats considèrent le plus souvent qu'il faut réaliser à l'intérieur du territoire national les infrastructures nécessaires pour assurer à chaque région, de manière équitable, un accès à l'eau. A l'extrême, dans les pays où la contrainte hydrologique est très forte, on estime plus sage de transférer l'eau que de déplacer les populations des zones trop arides. Rares sont les régimes qui ont tenté une politique inverse ; l'Ethiopie de Mengistu en est un exemple, avec les conséquences que l'on sait.

L'approche technocratique a été poussée jusqu'à la caricature dans l'ex-URSS où des projets pharaoniques

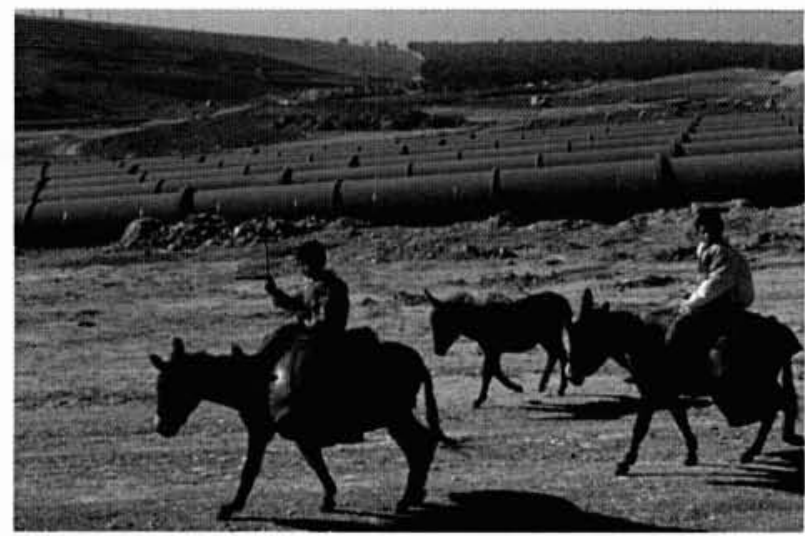

Conduites forcées au Maroc dans un paysage désertique. (Photothèque EDF - photo Marc Morceau) 
ont été réalisés (gigantesques transferts d'eau pour l'irrigation de l'est de l'Ukraine) ou envisagés (dérivation des fleuves sibériens vers l'Asie centrale, du Danube vers la Crimée), sans considération des incidences sur l'environnement. Mais ce serait une simplification abusive que de balayer comme vestiges de l'économie planifiée tous les " mégaprojets" mis en œuvre dans des pays aux régimes très divers :

- I'Espagne (Plan Hidrologico Nacional),

- I'Afrique du Sud (Lesotho Highlands Project),

- la Turquie (projet GAP pour l'irrigation de 1700000 ha dans le sud-est anatolien, le plus grand projet hydraulique en cours dans le monde : 32 milliards de \$ US),

- la Libye (Great Manmade River, 2000 km de conduites de 2 à $4 \mathrm{~m}$ de diamètre pour transférer vers la côte les eaux souterraines fossiles du désert libyen : 25 milliards de S US),

- sans oublier la France, avec les Sociétés d'Aménagement Régional créées dans les années 50 autour de grands projets tels que celui du Bas Rhône-Languedoc.

Tous ces aménagements sont localement justifiés par une volonté de créer ou maintenir des emplois dans des régions à économie agricole. Dans les trois premiers exemples au moins, les promoteurs se défendent, chiffres à l'appui, pour montrer que les préoccupations de développement durable ont été prises en considération. C'est ainsi que, d'après les études publiées par le gouvernement turc, le projet GAP, après achèvement vers 2005 , ne distraira que $12 \%$ des ressources en eau annuelles du bassin Tigre-Euphrate considéré à son embouchure.

Il faut reconnaître que, si les contraintes d'environnement ont bien été prises en compte dès les premières étapes de leur conception, ces grands projets hydrauliques ont l'avantage de permettre une maîtrise des ressources en eau face à la demande.

Ils sont probablement moins dommageables qu'une pression diffuse et anarchique des usagers de l'eau sur les écoulements naturels, sous la forme d'une multiplication de prélèvements " au fil de l'eau " dans les rivières ou les aquifères, conduisant à leur tarissement en période de sécheresse. La situation de certaines vallées du sud-ouest de la France au cours des étés 1989 - 1990, en est l'illustration.

\section{III — LA THÈSE GÉOSTRATÉGIQUE}

S’appuyant également sur des considérations de sécurité nationale et notamment d'autosuffisance alimentaire, des analystes ont mis l'accent sur les conflits réels ou potentiels entre Etats riverains des bassins du Jourdain, du Tigre/Euphrate, du Nil... mais aussi du Colorado, du Danube.

Une vision catastrophiste de ces situations a été largement développée dans les médias. Elle est fondée notamment sur le fait qu'il n'existe ni règles de droit international reconnues, ni organisation capable de les faire respecter. C'est une idée communément admise que "l'eau sera le motif des guerres du $3^{\mathrm{e}}$ millénaire".
Plusieurs communications présentées à Montréal prennent à contre-pied ces sinistres perspectives, et il est remarquable que beaucoup proviennent d'experts originaires du Moyen-Orient.

L'histoire des 4 dernières décennies ne permet d'identifier que 9 conflits armés dont l'eau ait été un des mobiles principaux ; la plupart sont restés d'ampleur très limitée. Pendant cette même période, 141 traités ont été signés sur la gestion concertée de ressources transfrontalières (A. Wolf - 1997).

La guerre est une issue peu vraisemblable aux conflits sur l'eau, pour plusieurs bonnes raisons :

- au plan stratégique, il est très difficile à un Etat agresseur de maîtriser l'usage de l'eau en dehors de ses frontières, sinon par une occupation permanente du terrain : - au plan économique, le coût d'un conflit armé est souvent beaucoup plus lourd que celui de ressources de substitution (stockage des eaux de saison humide, dessalement d'eau de mer...) ;

- les Etats riverains ont souvent beaucoup d'avantages à espérer d'une attitude coopérative, permettant par exemple de débloquer des projets d'aménagement bilatéraux profitables pour les deux parties : barrages, hydroélectricité, navigation...

Il est significatif que le traité indo-pakistanais sur l'Indus ait pu être signé en 1960 entre deux pays en situation d'extrême tension, et qu'il n'ait jamais été remis en cause à l'occasion des 3 conflits armés qui ont opposé ces deux nations depuis 40 années.

De même, le comité de Mékong a fonctionné régulièrement depuis 1957 dans une région fort troublée.

Enfin, la rapidité avec laquelle a été conclu en 1994 l'accord israélo-jordanien sur le Jourdain a montré que dès lors que les Etats avaient la volonté de s'entendre, l'eau ne constituait pas un enjeu de conflit insurmontable.

\section{IV — LA THÈSE ÉCONOMIQUE : LES MARCHÉS DE L'EAU}

L'eau est reconnue comme un bien économique. L'idée que des instruments à caractère économique peuvent être efficaces pour orienter les décisions individuelles des usagers dans le sens de l'intérêt collectif était déjà sousjacente à la loi sur l'eau de 1964 en France, mettant en place un système de redevances et d'incitations sur le " bon usage de l'eau ", géré par les Agences de Bassin.

Plus récemment, est apparu le concept de " marchés des droits d'eau ", développé notamment par les économistes de la Banque Mondiale. Pour réguler la répartition des eaux entre les divers usages, au sein d'un même bassin, il suffirait en économie libérale d'autoriser des transactions entre agriculteurs, municipalités et industriels portant sur les droits de prélèvement d'eau dans le milieu naturel, et de détacher ces droits de la propriété du sol. Le Chili a essayé de mettre en application cette doctrine, mais l'expérience est trop récente pour que l'on puisse se prononcer sur ses résultats. 
A l'échelle mondiale, il est clair que le concept de " marché global de l'eau " est vide de sens ; contrairement à l'énergie, l'eau ne peut être transportée économiquement sur de longues distances. Mais certains évoquent maintenant le " marché de l'eau virtuelle ". Le commerce mondial de produits agricoles est de fait la réponse à l'insuffisance de ressources en eau des pays confrontés à la pénurie. Il est beaucoup plus économique et " soutenable " dans une perspective à long terme, d'importer une tonne de blé (1000 F au cours mondial) que de la produire dans un pays aride, en utilisant environ $1000 \mathrm{t}$ d'eau précieuse, parfois prélevée sur des réserves fossiles, au prix de projets pharaoniques qui immobilisent les capacités d'investissement locales. Certains analystes comme H. Shuval estiment que le concept de l'autosuffisance alimentaire pays par pays est dépassé.

Ils font observer que certains pays y ont renoncé (c'est le cas d'Israël, ou même de l'Egypte qui, bien que mieux dotée en eau, importe $50 \%$ de ses besoins en denrées alimentaires).

Selon eux, la revendication à l'autosuffisance alimentaire est non seulement antiéconomique mais dangereuse car elle conduit à une impasse génératrice de conflits. On estime le besoin en eau par habitant, production agricole comprise, à $1800 \mathrm{~m}^{3} / \mathrm{an}$, alors que beaucoup de nations du Moyen-Orient disposent globalement de moins de $1000 \mathrm{~m}^{3} / \mathrm{an}$. En revanche, 100 à $150 \mathrm{~m}^{3} / \mathrm{an}$ par habitant sont suffisants pour assurer les besoins domestiques et industriels, avec un niveau convenable de confort et de développement économique. Seuls quelques pays assez peu peuplés (Libye, Etats du Golfe, îles...) sont en situation de "pénurie absolue " en dessous de ce seuil. Encore faut-il le considérer comme relatif car le dessalement d'eau de mer ou le retraitement de l'eau usée permettent de produire de l'eau douce à environ $1 \mathrm{USD} / \mathrm{m}^{3}$, coût acceptable pour les usages urbains et industriels dans des pays industrialisés.

\section{$V \square$ POUR UNE ANALYSE LUCIDE DES POSITIONS EN PRÉSENCE}

Evidemment, les thèses développées précédemment ne sont pas totalement dénuées d'arrière-pensées, inspirées par des intérêts économiques ou nationaux.

Par exemple, on peut être frappé de voir les tenants des thèses écologistes les plus radicales particulièrement actifs dans les pays nordiques, qui ne sont pas menacés par la rareté de l'eau, ou encore au Canada et aux USA, grands exportateurs de produits agricoles. L'abandon de projets d'irrigation dans les pays du Sud tirerait évidemment à la hausse les cours des céréales.

La dramatisation des enjeux stratégiques liés à l'eau peut relever d'une certaine forme de propagande, ou pour le moins de pressions des lobbies intéressés par le développement des budgets militaires dans les régions sensibles.

L'accentuation des risques de pénurie peut aussi procéder d'un discours d'auto-justification de certaines institutions œuvrant dans le domaine de l'eau. Si la sensibilisation du

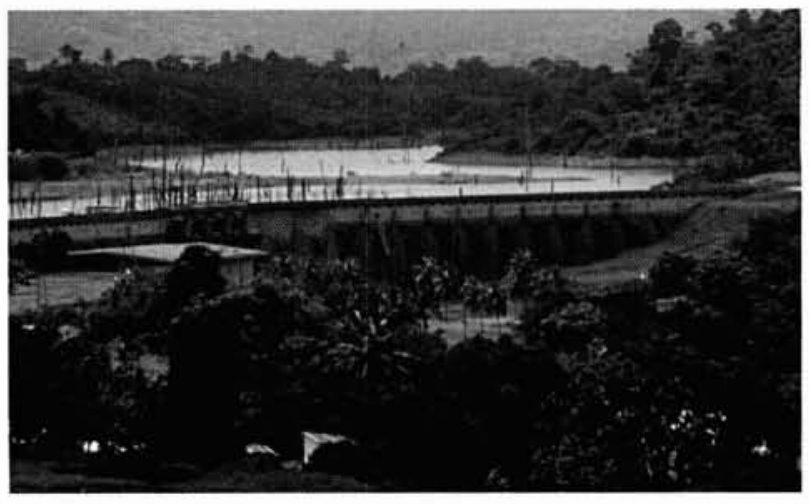

Paysage verdoyant de Côte d'Ivoire.

(Photothèque EDF - photo Marc Morceau)

public à la protection de l'eau est un objectif louable, elle prend parfois des formes caricaturales : une carte des régions du monde soumises à des risques de tensions futures sur l'eau, présentée à Montréal, faisait apparaître... le Canada comme un pays risquant de connaitre un stress hydrique. Une communication faisait état des efforts engagés pour réduire les consommations d'eau urbaine à... Terre Neuve.

Des amalgames ou ambiguiités entachent trop souvent la présentation au grand public des enjeux sur les ressources en eau. Beaucoup de déclarations commencent par l'évocation du milliard d'hommes qui n'ont pas accès à une eau potable sanitairement acceptable. Ce fait bien réel ne relève pas de l'insuffisance des ressources, mais du manque d'équipements pour les traiter et les distribuer. La répartition de ce milliard d'hommes dans le monde coïncide avec la carte de la pauvreté, non avec celle de l'aridité. Bien plus, les mêmes populations souffrent assez souvent d'excès d'eau saisonniers, comme dans les bidonvilles de Calcutta ou de Bombay.

On a trop tendance aussi à confondre mobilisation et consommation de l'eau. Dans un même bassin versant, l'eau peut servir à plusieurs usages en cascade si elle est correctement épurée. Sans même parler de réutilisation directe des eaux usées, la réutilisation " au fil de l'eau " est devenue un fait dans les bassins à grande densité humaine, comme celui de la Seine, ou de la Tamise où l'on considère qu'en été l'eau est utilisée 3 fois.

Les thèses à fondement économique peuvent avoir un effet salutaire en ramenant un peu de sérénité et de rigueur dans des débats souvent trop subjectifs. Elles ont cependant leurs limites : le renoncement à l'autosuffisance agricole peut être concevable au plan de la sécurité alimentaire, mais il pose souvent le problème de l'emploi dans des populations où l'agriculture occupe encore plus de $50 \%$ des actifs. Il implique une conversion sociale qui ne pourra se réaliser qu'en plusieurs générations.

Faire la synthèse de ces positions contradictoires est une tâche impossible et probablement vaine. C'est au plan local, à l'échelle des bassins versants beaucoup plus qu'à l'échelle planétaire, qu'il faut répondre aux défis de l'eau, qui sont extrêmement divers d'une région à l'autre. 